\title{
Analysis of Dose Calculation Accuracy in Cone Beam Computed Tomography with Various Amount of Scattered Photon Contamination
}

\author{
Keisuke Usui' ${ }^{1}$, Koichi Ogawa², Keisuke Sasai ${ }^{1}$ \\ ${ }^{1}$ Department of Radiation Oncology, Juntendo University, Tokyo, Japan \\ ${ }^{2}$ Faculty of Science and Engineering, Hosei University, Tokyo, Japan \\ Email: k-usui@juntendo.ac.jp
}

How to cite this paper: Usui, K., Ogawa, K. and Sasai, K. (2017) Analysis of Dose Calculation Accuracy in Cone Beam Computed Tomography with Various Amount of Scattered Photon Contamination. International Journal of Medical Physics, Clinical Engineering and Radiation Oncology, 6, 233-251. https://doi.org/10.4236/ijmpcero.2017.63022

Received: May 17, 2017

Accepted: July 3, 2017

Published: July 6, 2017

Copyright $\odot 2017$ by authors and Scientific Research Publishing Inc. This work is licensed under the Creative Commons Attribution International License (CC BY 4.0).

http://creativecommons.org/licenses/by/4.0/

c) (†) Open Access

\begin{abstract}
Cone-beam computed tomography (CBCT) images have inaccurate CT numbers because of scattered photons. Thus, quantitative analysis of scattered photons that affect an electron density (ED) curve and calculated doses may be effective information to achieve CBCT-based radiation treatment planning. We quantitatively evaluated the effect of scattered photons on the accuracy of dose calculations from a lung image. The Monte Carlo method was used to calculate $\mathrm{CBCT}$ projection data, and we made two calibration curves for conditions with or without scattered photons. Moreover, we applied cupping artifact correction and evaluated the effects on image uniformity and dose calculation accuracy. Dose deviations were compared with those of conventional $\mathrm{CT}$ in conventional and volumetric intensity modulated arc therapy (VMAT) planning by using $\gamma$ analysis and dose volume histogram (DVH) analysis. We found that cupping artifacts contaminated the scattered photons, and the $\gamma$ analysis showed that the dose distribution was most decreased for a scattered photon ratio of $40 \%$. Cupping artifact correction significantly improved image uniformity; therefore, ED curves were near ideal, and the pass rate results were significantly higher than those associated with the scattered photon effect in $65.1 \%$ and $78.4 \%$ without correction, $99.5 \%$ and $97.7 \%$ with correction, in conventional and VMAT planning, respectively. In the DVH analysis, all organ dose indexes were reduced in the scattered photon images, but dose index error rates with cupping artifact correction were improved within approximately $10 \%$. CBCT image quality was strongly affected by scattered photons, and the dose calculation accuracy based on the $\mathrm{CBCT}$ image was improved by removing cupping artifacts caused by the scattered photons.
\end{abstract}

\section{Keywords}

Cone Beam Computed Tomography, Scattered Photon, Dose Calculation, 
Cupping Artifact

\section{Introduction}

A kilo-voltage cone-beam computed tomography (CBCT) system mounted on a linear accelerator has become available for image guided radiotherapy. The main purpose of $\mathrm{CBCT}$ is to improve the accuracy of target localization in radiation therapy [1]. CBCT can verify not only tumor position but also tumor regression or progression on each radiation treatment day. Furthermore, if CBCT images are also available for accurate dose calculation, a planned dose distribution can be verified every treatment day [2]. Consequently, we can avoid excessive radiation doses and calculate a dose distribution that considers modification of the target before or after each radiation therapy. Therefore, we can use CBCT images for adaptive radiation therapy (ART) [3]. However, CBCT images are degraded by scattered photons and cannot provide an accurate CT number; hence, such images cannot be used directly for accurate dose calculation.

In previous studies, degradation of accuracy in calculated dose distribution has been shown when original CBCT numbers were used for dose calculations; therefore, replacement of CBCT numbers with conventional CT numbers has been suggested [4] [5] [6] [7] [8]. Richter et al. studied several conversion methods of СВCT numbers measured under different conditions by using two phantoms and 33 patients [4], and Hu et al. used an approach to make a conversion curve for each patient by using CBCT images acquired on the day of the planning CT [5]. These proposed methods can improve the accuracy of pixel value conversion between the СBCT and conventional CT number [4]. However, an appropriate conversion curve depends on the treatment conditions, such as data acquisition modes and amount of scattered photons. Consequently, several conversion curves for different data acquisition modes or target sizes, including patients' sizes and regions, are required for improvement of CBCT number conversion accuracy. Using a method with СBCT density override, Fotina et al. investigated $\mathrm{CBCT}$ and conventional $\mathrm{CT}$ number adjustment techniques by using image segmentation and an ROI mapping method [7]. These approaches were most robust against CBCT image quality and shown to provide good results; however, they were adversely affected by the differences in the accuracy of structure segmentation and organ deformation between the CBCT and CT image [9].

If we could reconstruct a $\mathrm{CBCT}$ image by using accurate Hounsfield unit values, we could improve the accuracy of dose calculations by using a quantitative image value without having to use several CBCT number replacement methods. Therefore, this approach can save time in the CBCT based re-planning and adaptive planning process. However, image quality of CBCT was affected by the scattered photon and the beam hardening artifact. In previous studies on scattered photon correction methods for CBCT images, several algorithmic methods 
have been proposed for estimating and correcting scattered photons in the CBCT projection data. Zhu et al. proposed the blocker-based method that allows approximate characterization of the scattered photons in the shadow of the blocker [10]. Moreover, Maltz et al. [11] and Sun et al. [12] investigated the scatter kernel deconvolution studies, which used Monte Carlo (MC) simulation, and Thing et al. [13] applied these methods to patient-specific scatter correction. MC simulation offers a potentially accurate method for scatter estimation, but applying MC-based correction to a specific CBCT image is limited by the high computational complexity associated with the large simulation time and the need for an acceptably low noise level [14].

Adequate level of scattered photon reduction in the CBCT image for accurate dose calculation can be a goal of these CBCT image improvement methods, however, knowledges of amount for reduction of scattered radiation to achieve dose calculation with acceptable precision accuracy using $\mathrm{CBCT}$ has not been known. Thus, quantitative analysis of the scattered photons that affect a calibration curve of CBCT number to electron densities and calculated doses may be effective information for achieving CBCT-based radiation treatment planning. This type of investigation is possible to reveal the $\mathrm{CBCT}$ image quality for accurate dose calculations in patients.

In this study, we quantitatively evaluated the effect of scattered photons on the accuracy of dose calculations by using CBCT images. We simulated the CBCT projection data of an electron density (ED) phantom by using the MC method and made calibration curves of CBCT pixel numbers to several electron densities, including various ratios of the scattered photons. Similarly, we calculated patient lung images and converted CBCT pixel numbers to electron densities by using these calibration curves. Additionally, we determined the relationship between the accuracy of calculated patient dose and the amount of the scattered photons in $\mathrm{CBCT}$ images. Moreover, the effects of cupping artifact correction on $\mathrm{CBCT}$ images and on image uniformity were also investigated along with the dose calculation accuracy for CBCT images with cupping artifact correction.

\section{Material and Methods}

\subsection{Data Acquisition of the ED Phantom Image and Curves}

The MC method was used to calculate CBCT projection data of an ED phantom. This calculation system was in-house program, and we calculated transport of only photons with kilovolt energy using a random number generator with the Mersenne twister for calculating probability of interactions [15]. We simulated a data acquisition system by using a $40.0 \times 30.0 \mathrm{~cm}^{2}$ flat-panel detector. Figure 1 shows the geometry of the CBCT projection data acquisition, and Table 1 shows the data acquisition conditions. In this simulation, the number of photons used was $0.1 \mathrm{M}$, and we emitted these photons in the cone direction from a point source that was located just under the center of the detector surface. The distances from the source to the rotation axis and the detector were $100 \mathrm{~cm}$ and 150 $\mathrm{cm}$, respectively. The CBCT projection images were acquired by using an 


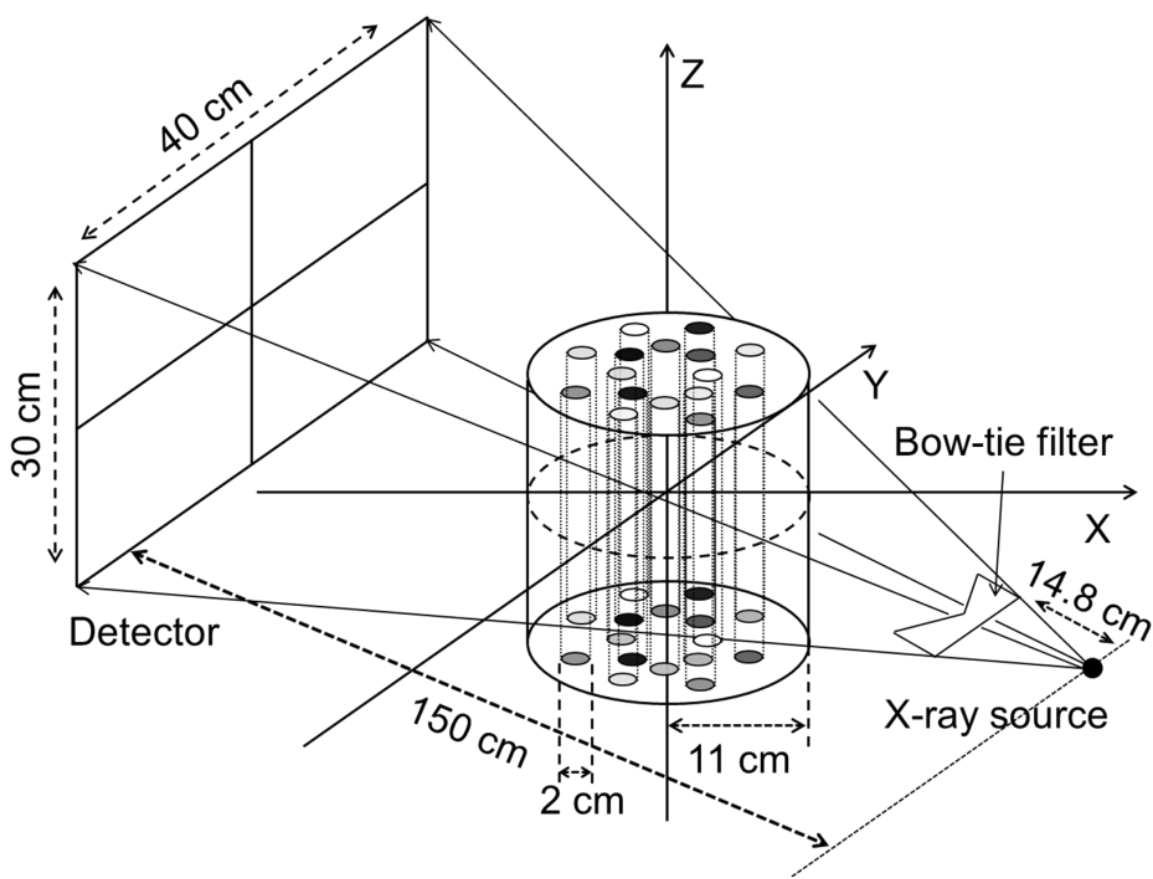

Figure 1. Geometry of the CBCT system with ED phantom.

Table 1. Conditions of the projection data acquisition using the MC method.

$\begin{array}{cc}\text { Tube voltage } & 100 \mathrm{kV} \\ \text { Number of photons } & 100,000 \\ \text { Data acquisition angle } & 360^{\circ} \\ \text { Number of projections } & 180 \\ \text { Detector matrix } & 400 \times 300 \text { pixels } \\ \text { Pixel size } & 1.0 \times 1.0 \mathrm{~mm}^{2} \\ \text { Calculation grid } & 1.0 \mathrm{~mm} \\ \text { Cutoff energy } & 30 \mathrm{keV} \\ \text { Random generator } & \text { Mersenne twister } \\ \text { Recon algorithm } & \text { FBP } \\ \text { Recon filter } & \text { Shepp \& Logan }\end{array}$

$\mathrm{x}$-ray tube with a voltage of $100 \mathrm{kV}$, and we acquired 180 projection images of $2^{\circ}$ each over $360^{\circ}$. Regarding the interactions of photons with materials, we considered compton scattering, coherent scattering, and the photoelectric effect, and we defined the scattered photons as the photons involved in these interactions. The calculation accuracy of this MC simulation confirmed that counts of attenuated photons and energy spectrum coincide with the theoretically calculated value with an accuracy within $1.0 \%$.

For the CBCT pixel number conversion curves of the ED phantom, which consisted of the ten density specification materials (air, lung, adipose, breast, water, soft tissue, muscle, liver, mineral bone, and cortical bone) were reconstructed with the MC projection data. The diameter of the ED phantom was 22 
$\mathrm{cm}$, and each material was $2.0 \mathrm{~cm}$. The composition of the ED phantom and the details of each material are shown in Figure 2 and Table 2 [16]. This ED phantom image was reconstructed by using a filtered-back projection method with a Shepp and Logan filter [17]. The size of an image matrix was $400 \times 400$ pixels $\left(40 \times 40 \mathrm{~cm}^{2}\right)$ and the slice thickness was $1.0 \mathrm{~mm}$. In this study, the reconstructed image was positioned on the central axis of the beam line; thus, we could not consider the effect of the cone beam angle.

We then made two types of calibration curves of CBCT pixel values to several electron densities: one was a condition that included various ratios of scattered photons and another one without scattered photons, which curve means equivalent one acquired by the multi-slice CT (MSCT). Because, detector is strictly collimated in MSCT system, thus scattered photon contaminations are very small. The ratios of the scattered photon contaminations for each curve were successively reduced by $20 \%$ in $100 \%-20 \%$ from the ratio used in the previous calibra-

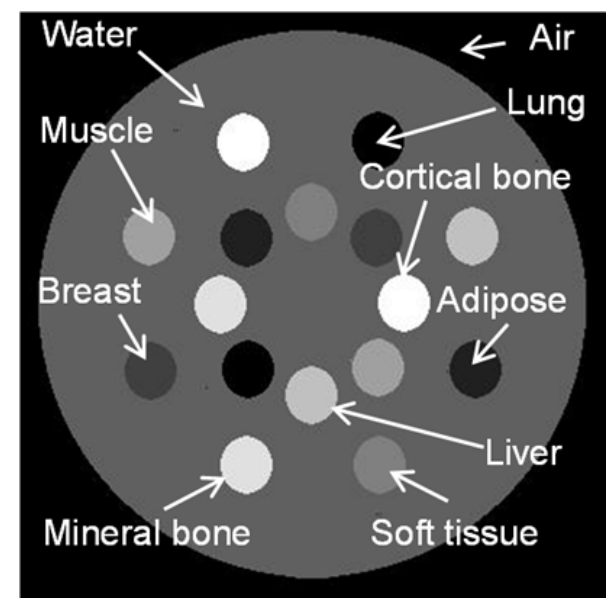

Figure 2. Composition of the electron density phantom. This phantom consisted of the ten electron density specification materials (air, lung, adipose, breast, water, soft tissue, muscle, liver, mineral bone, and cortical bone).

Table 2. Mass density and relative electron density of each material in the ED phantom.

\begin{tabular}{ccc}
\hline & Mass density $\left(\mathrm{g} / \mathrm{cm}^{3}\right)$ & Electron density \\
\hline Air & 0.00 & 0.00 \\
Lung & 0.28 & 0.29 \\
Adipose & 0.94 & 0.93 \\
Breast & 0.98 & 0.96 \\
Water & 1.00 & 1.00 \\
Soft tissue & 1.02 & 1.01 \\
Muscle & 1.05 & 1.02 \\
Liver & 1.10 & 1.06 \\
Mineral bone & 1.15 & 1.10 \\
Cortical bone & 1.82 & 1.69 \\
\hline
\end{tabular}


tion curve, moreover $10 \%$ and $5 \%$ - $1 \%$ scattered photon contaminations curves were obtained. Additionally, we set ten circular regions of interest (ROIs), and obtained the mean pixel value in the reconstructed image. The size of the circular ROI was 10 pixels. Using these conversion curves, we associated each ED value with the corresponding CBCT pixel value.

\subsection{Lung Image Data Acquisition}

To compare the effect of image quality on the accuracy of the calculated doses from the CBCT image, we acquired projection data of the lung images treated by radiation therapy for lung cancer by using the MC method under the same conditions in section 2.1, and reconstructed the lung images under each condition by including the scattered photons corresponding to each calibration curve. In this simulation, the MSCT of the lung image, which was acquired in previously, was divided into three organs (lungs, bones, and soft tissues), and linear attenuation coefficients were obtained from these three areas. Projection data of this lung image attenuated by each region according to the linear attenuation coefficients were calculated and counted with the MC method.

For these lung image studies, dose distributions were calculated from CBCT images after conversion of the CBCT pixel values by using the calibration curve. As a reference dose distribution, the MSCT planning was calculated by using the standard CT value calibration curve acquired from the MSCT system.

\subsection{Image Uniformity and Cupping Artifact Correction}

Beam hardening and scattered photons each reduced the measured attenuation coefficients. As a result, beam hardening and scattered photons produced a common artifact known as the cupping artifact [14] [18]. In this paper, using the MC method, we simulated the projection data in a uniform water cylinder, which had a diameter the same as that of the ED phantom, and evaluated the uniformity and the profile of the reconstructed image depending on the amount of the scattered photons. The conditions of the MC method are shown in Table 1. A parameter used to evaluate the image uniformity $\left(t_{\text {cup }}\right)$ was calculated according to Equation (1), and then we acquired the attenuation coefficient from a center $\left(\bar{\mu}_{\text {center }}\right)$ and edge $\left(\bar{\mu}_{\text {edge }}\right)$ of the cylinder image.

$$
t_{\text {cup }}[\%]=100 \times \frac{\bar{\mu}_{\text {edge }}-\bar{\mu}_{\text {center }}}{\bar{\mu}_{\text {edge }}}
$$

To correct the cupping artifact on the CBCT reconstructed image, a simple correction method assuming a uniform water object was used. In this method, we calculated ideal projection data $(R j)$ with an effective spectrum $(E m)$ from the transmission distance $(L(j))$ of the water object in each projection, as shown in Equation (2). In cupping artifact correction, pixel values with the cupping artifact were modified according to these ideal projected dates, therefore, both the effect of scattered photons and beam hardening were corrected with this method. Then, we created cupping artifact correction maps in every ratio of the 
scattered photons, and the ED phantom image and patient lung image with cupping artifact were modified using these correction maps in each scattered photon classes.

$$
R j=I \exp \left[-\mu_{w}\left(E_{m}\right) \cdot L(j)\right]
$$

I: number of photons

$\mu_{w}:$ attenuation coefficient with $E m$

Moreover, the ED phantom image and the lung image were corrected for the cupping artifact, and the accuracies of the calculated doses were evaluated. In correction of the lung image, the inside of the lung image contour was replaced with water material, and similarly, correction maps in every ratio of the scattered photon contaminations were created, and the cupping artifact in lung images were modified.

\subsection{Evaluation of Dose Calculation Accuracy}

The differences between the dose distributions calculated from the lung CBCT image with scattered photons and from the image without scattered photons were analyzed by using the $\gamma$ analysis. The criteria for the pass rate in the $\gamma$ analysis were $3 \%$ of the absolute dose and $\leq 3 \mathrm{~mm}$ agreement. In this evaluation, fivefield beam conventional planning and 1-arc volumetric arc therapy (VMAT) planning (coplanar, 6-MV x-rays) were calculated by using the Eclipse ver. 13.6 treatment planning system (Varian Medical Systems, USA), and the anisotropic analytical algorithm (AAA) and the Acuros XB algorithm were used for the dose calculation algorithm. The prescribed dose was $200 \mathrm{cGy}$ for $95 \%$ of the target volume, and each structural contour was common size and location in each plan. Moreover, the dose distributions were evaluated by using dose volume histogram (DVH) analysis, and dose differences among the scattered photons in this lung image were expressed as the $D_{\text {mean }}$ and $D_{95}$ in each target area and the $V_{20}$, $V_{5}, D_{\text {lcc }}$ and $D_{\text {mean }}$ in several organs at risk (OARs). In these evaluations, the differences between the dose distributions based on the MSCT image and those based on the CBCT images with scattered photons were analyzed. Therefore, the contour of the target organs and OARs, planning geometry, and number of monitor units remained unchanged.

\section{Results}

\subsection{Reconstruction Image and Conversion Curve in the ED Phantom}

Figure 3 shows the reconstructed image of the ED phantom with various percentages of scattered photons. These results showed the presence of low gray value areas (cupping artifact) in the center of the reconstructed images contaminating the scattered photons. Cupping artifact correction showed that image uniformity was significantly improved in each reconstructed image. Figure 4 shows the conversion curve between the CBCT pixel value and the ED value. These curves were generated from the ED phantom image data, and for con- 
struction of these curves, a total of ten different ROIs were used. As the scattered photons were reduced, the ED curves became closer to the ideal value (curve of the primary photons). Moreover, with cupping artifact correction, the slopes of

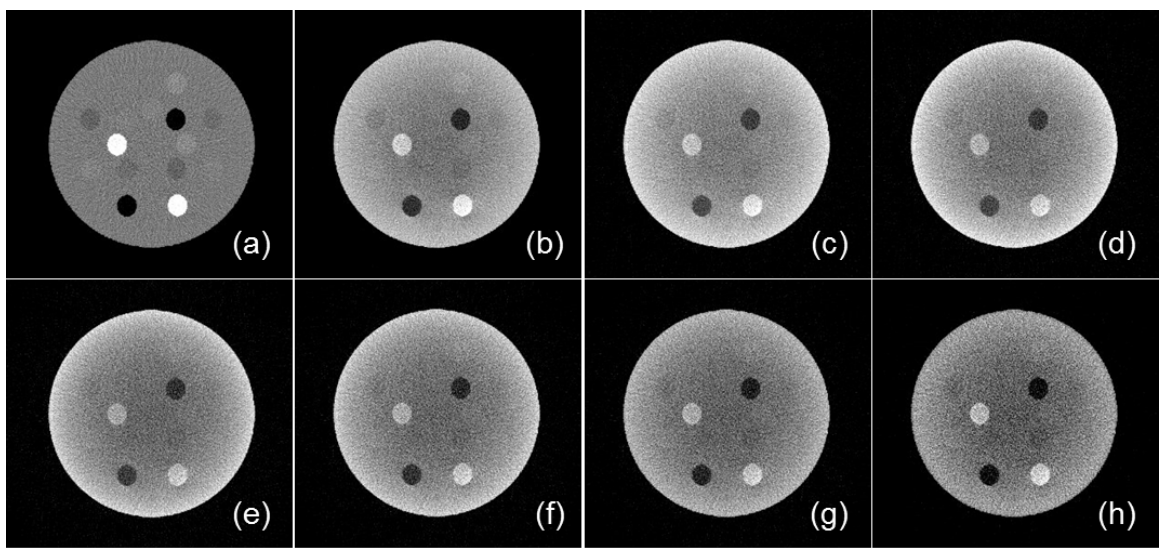

(i)

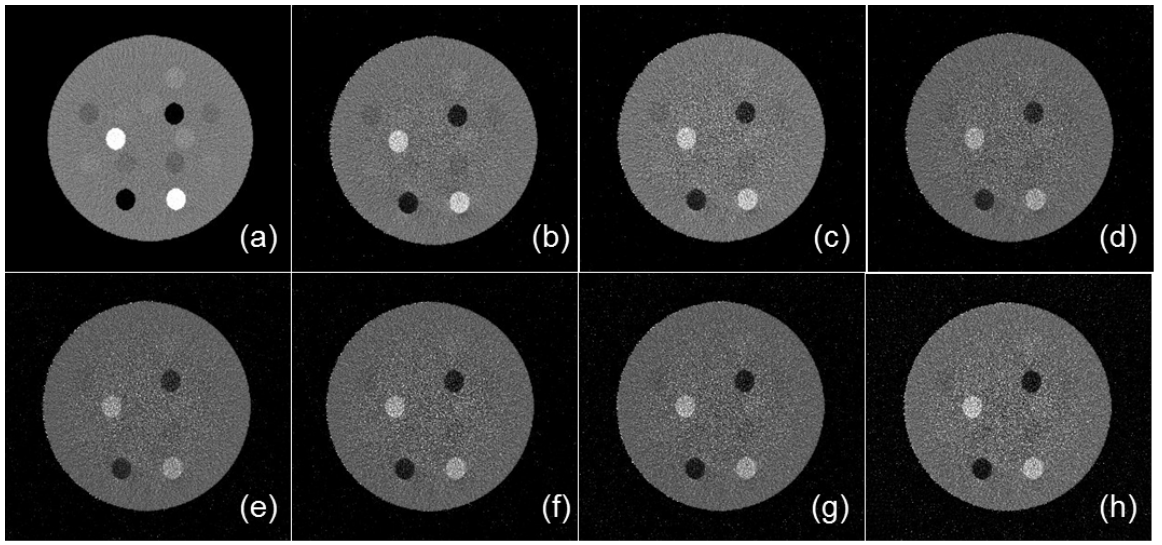

(ii)

Figure 3. Reconstructed images of the ED phantom. (a) was reconstructed by using only primary photons, and (b)-(h) were reconstructed by using scattered photons at different ratios; (b): 5\%, (c): 10\%, (d): 20\%, (e): 40\%, (f): 60\%, (g): 80\%, (h): 100\%, (i): without cupping artifact correction, (ii): with cupping artifact correction.

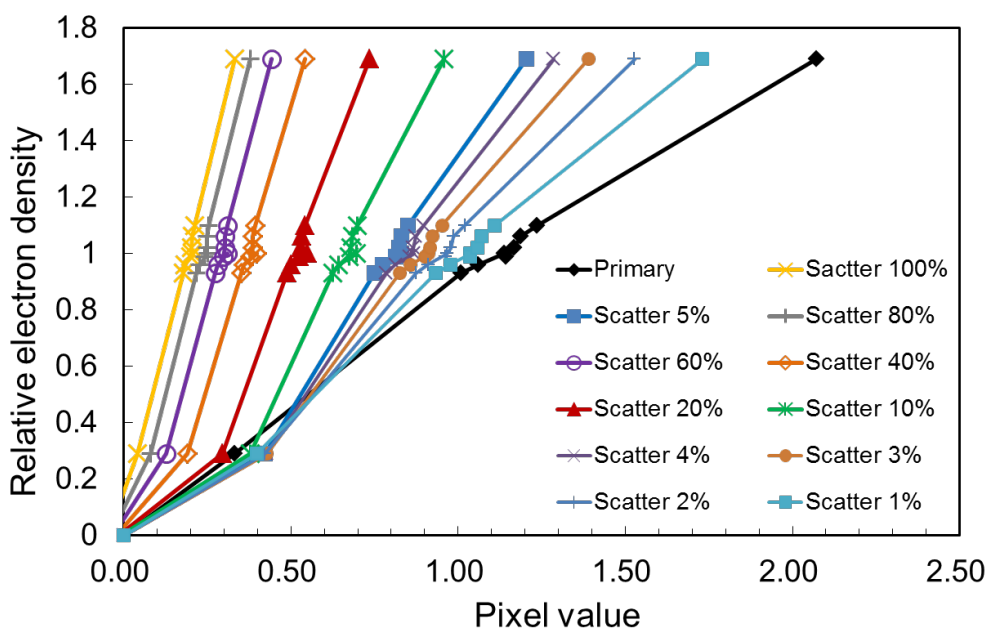

(i) 


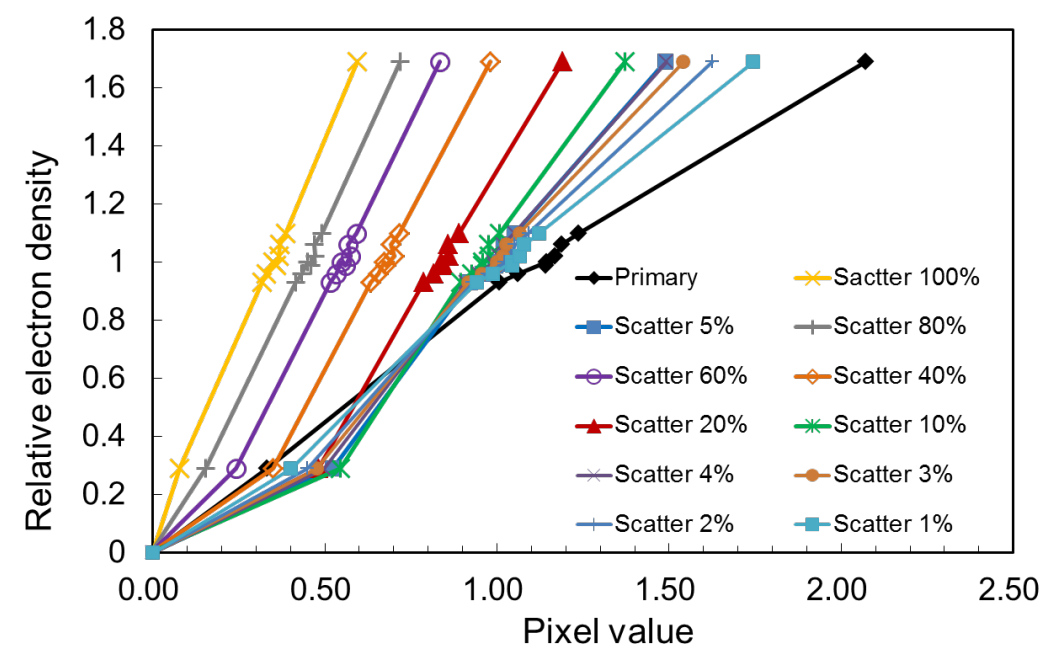

(ii)

Figure 4. ED curves consisting of primary and scattered photons. (i): without cupping artifact correction, (ii): with cupping artifact correction.

these curves approached the primary photon curve; thus, the differences between the ED values corresponding to the pixel values were reduced.

\subsection{Image Uniformity}

Figure 5 shows the results of the reconstructed image and the profile of the water cylinder phantom. Table 3 shows the results of the image uniformity of this phantom for different contaminating ratios of the scattered photons. Cupping artifacts appeared in the reconstructed image with scattered photons. Image uniformity was most degraded at a scattered photon contamination ratio of $40 \%$. On the other hand, when the scattered photon contamination ratio exceeded $40 \%$, the image uniformity slightly improved.

\subsection{Evaluation of Dose Distribution}

Figure 6 shows the dose distribution in the lung image calculated from the MDCT image. Figure 7 shows the dose distribution in the CBCT image for each ED curve without cupping artifact correction, and Figure 8 shows the dose distributions with cupping artifact correction. Subfigure 7, 8A shows conventional five-field planning, and subfigure 7, 8B shows 1-arc VMAT planning. The dose distribution in the primary photon image (a) is similar to that in the MSCT image (Figure 6(A)); on the other hand, the images (b)-(h) of the dose distributions for different scattered photon ratios without cupping artifact correction were different from those of the MSCT plan in terms of the shape of the isodose curves near the tumor. In the conventional planning (Figure $8(\mathrm{~A})$ ) and the VMAT planning (Figure 8(B)), these dose distributions were similar to those of the MSCT planning regardless of the amount of scattered photons.

Figure 9 shows the results of the $\gamma$ analysis of the calculated lung images. We compared the calculated dose distributions between the MSCT image and the CBCT image. Therefore, the pass rate of the $\gamma$ analysis shows the consistency of 

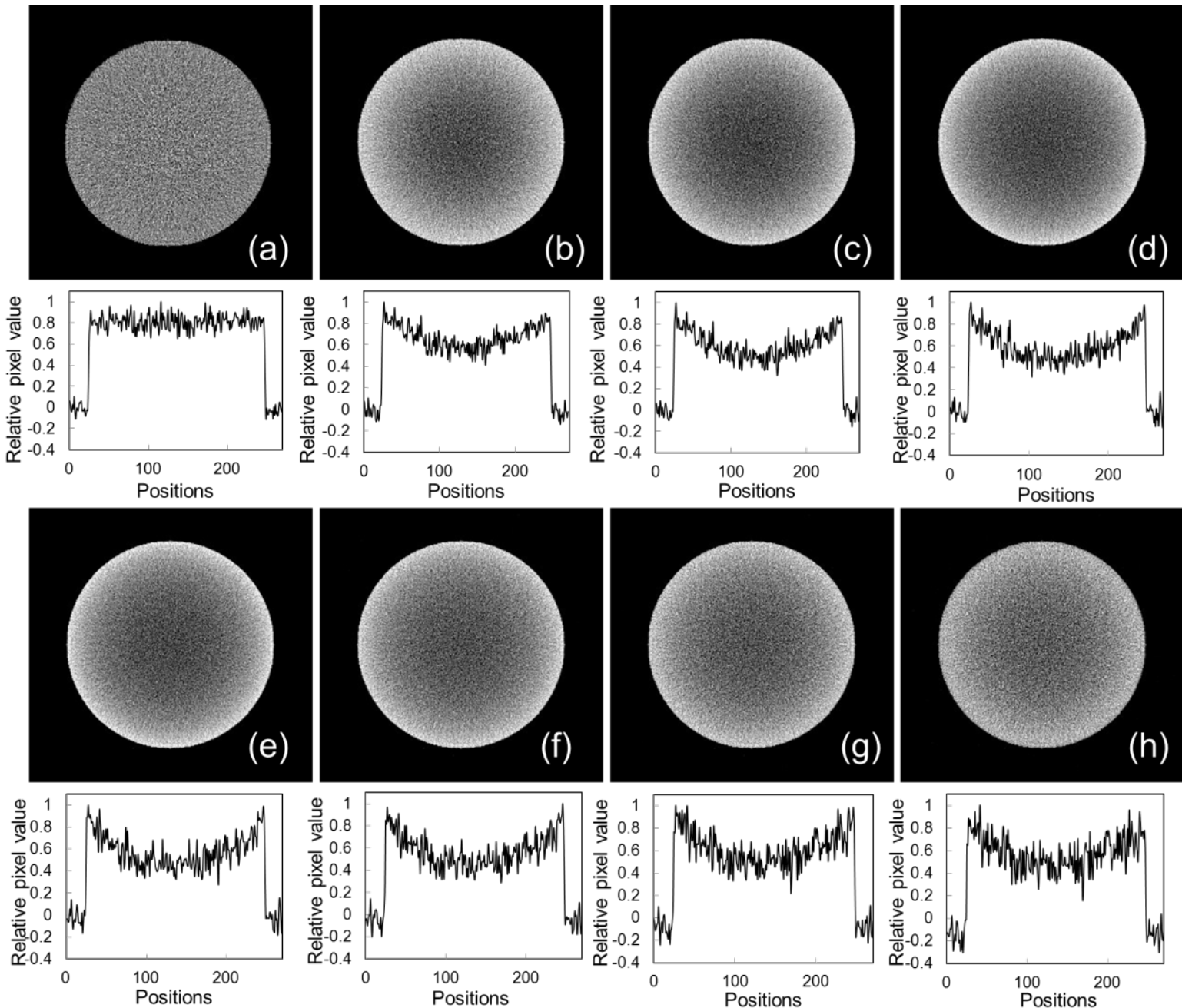

Figure 5. Reconstructed images and profiles of pixel values in a water cylinder phantom for each contaminating ratio of scattered photons. (a) was reconstructed by using only primary photons, and (b)-(h) were reconstructed by using scattered photons at different ratios; (b): 5\%, (c): 10\%, (d): 20\%, (e): 40\%, (f): 60\%, (g): $80 \%$, (h): $100 \%$.

Table 3. Image uniformity of the water cylinder phantom.

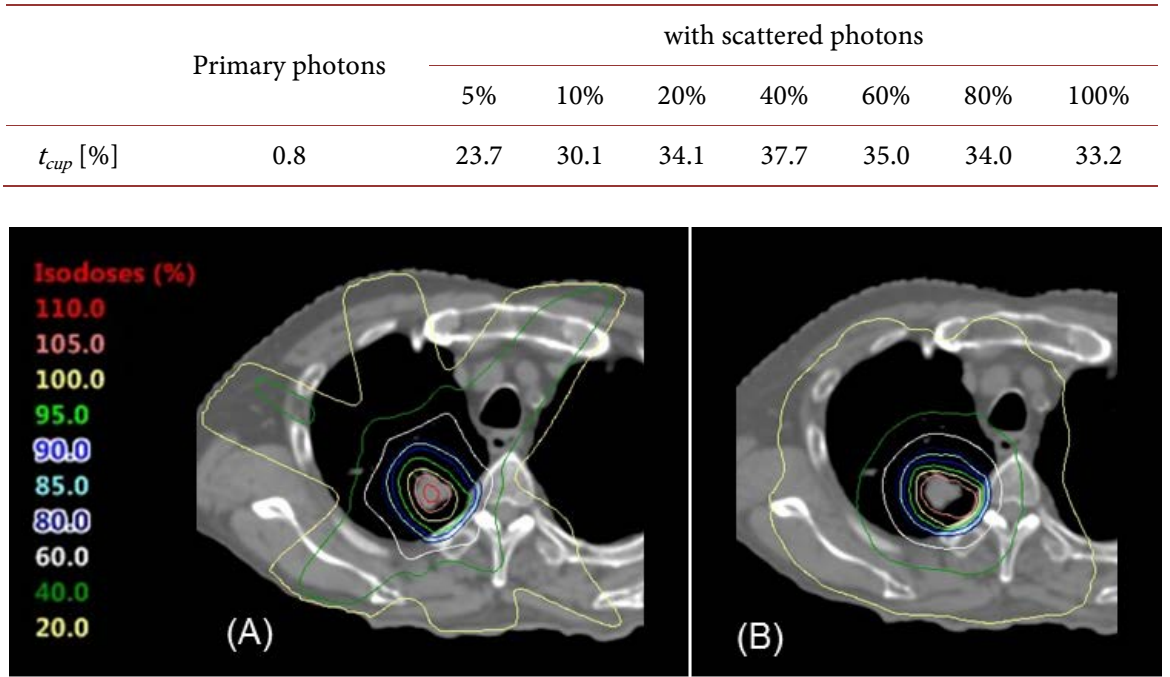

Figure 6. Dose distributions using the MDCT image. (A): conventional five-field planning, (B): 1-arc VMAT planning. 


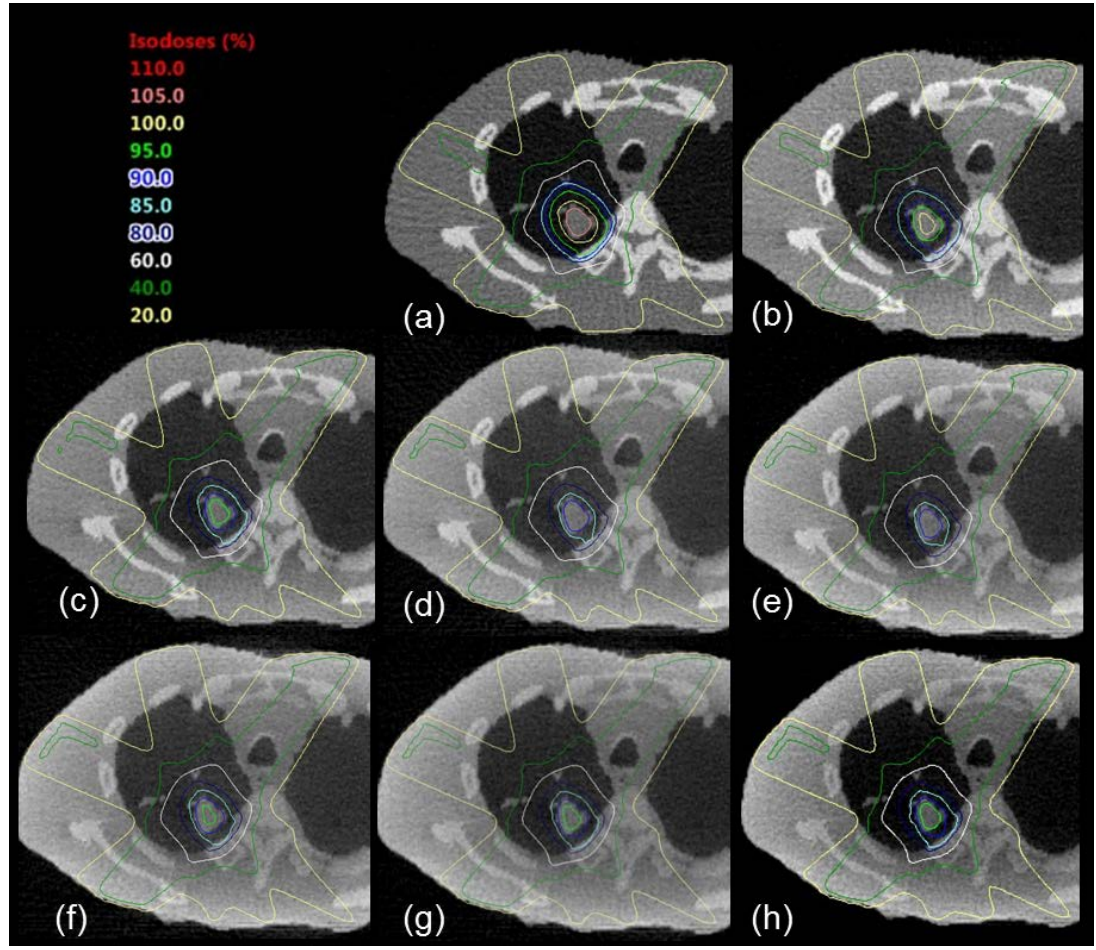

(A)

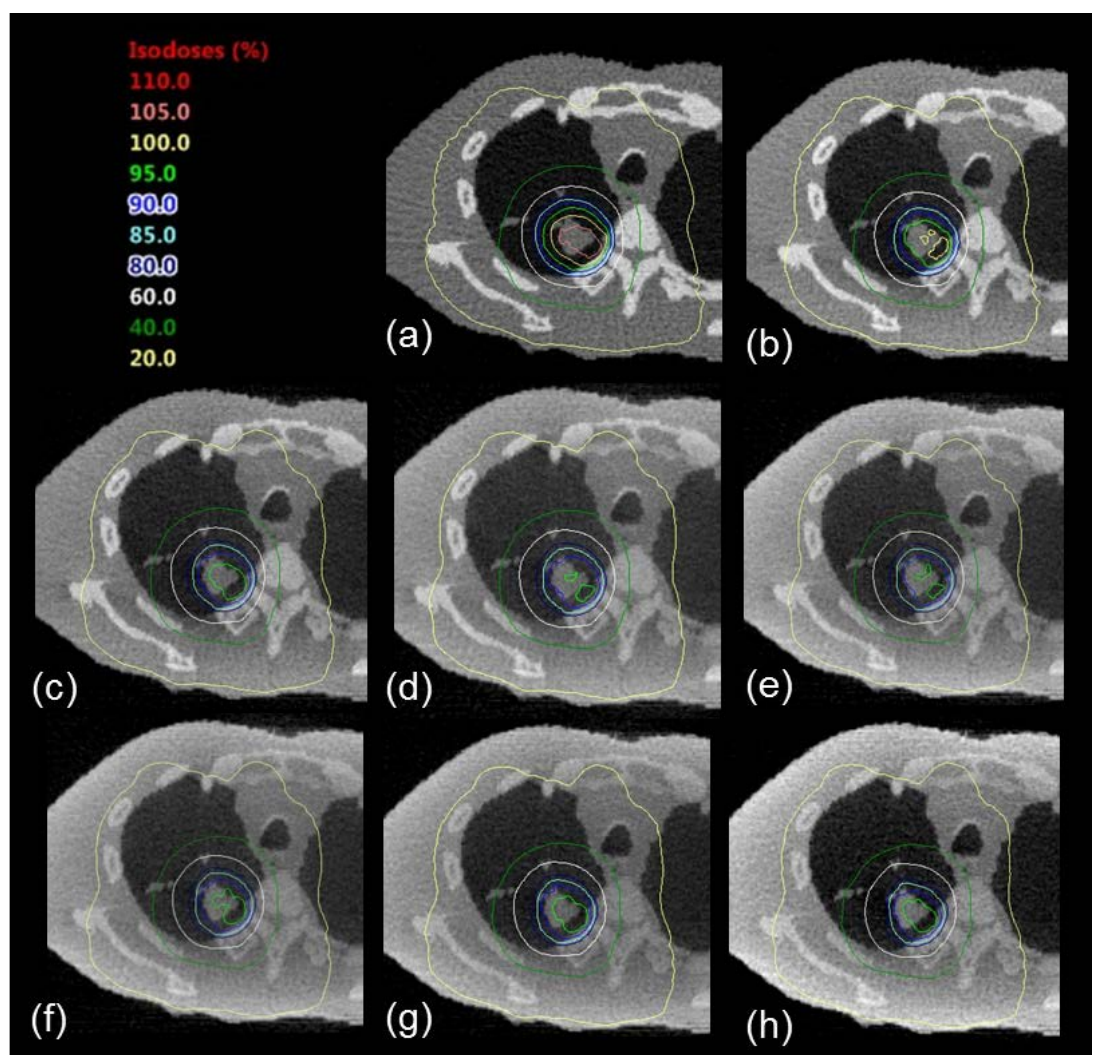

(B)

Figure 7. Dose distributions using the CBCT image without cupping artifact correction. These lung images were reconstructed by using scattered photons at different ratios; (a): only primary photons, (b): $5 \%$, (c): $10 \%$, (d): $20 \%$, (e): 40\%, (f): $60 \%,(g): 80 \%$, (h): $100 \%$ with scattered photons. (A): conventional five-field planning, (B): 1-arc VMAT planning. 


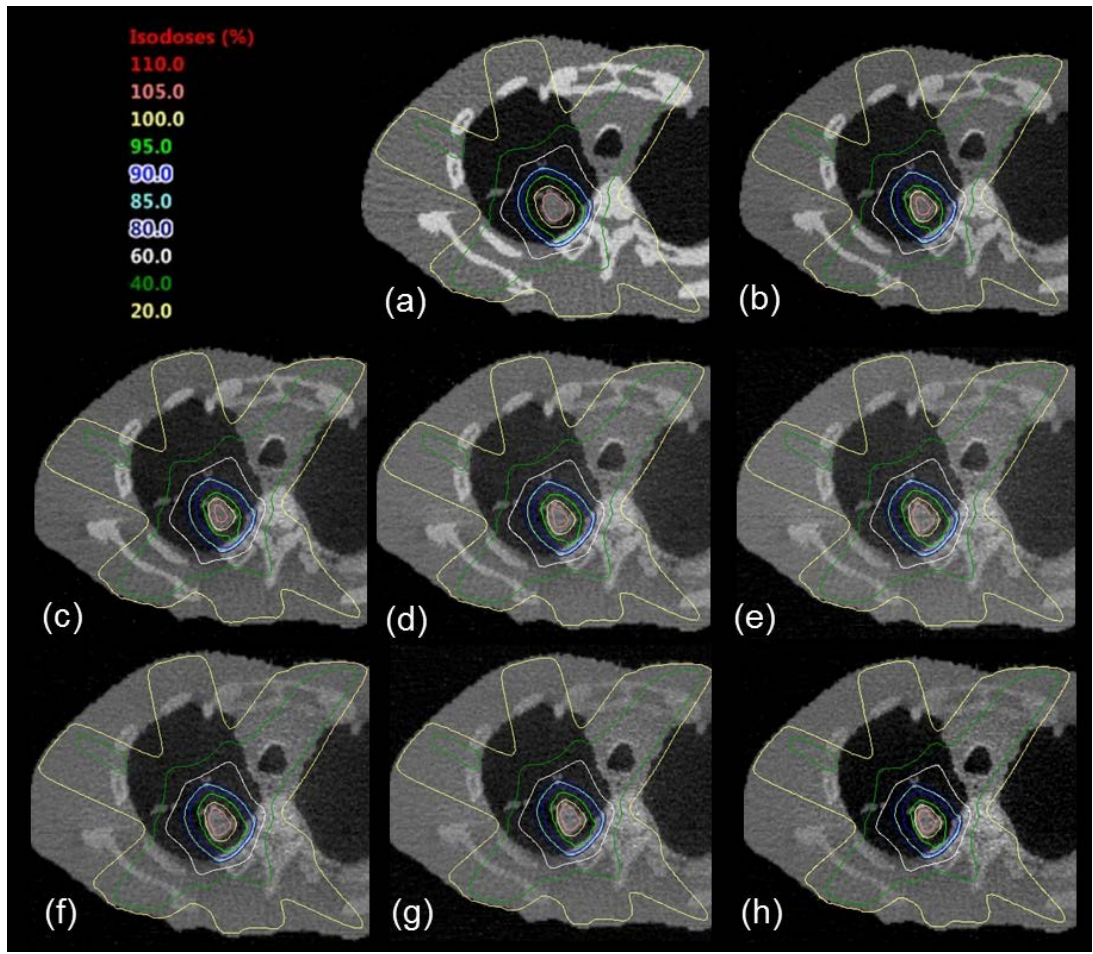

(A)

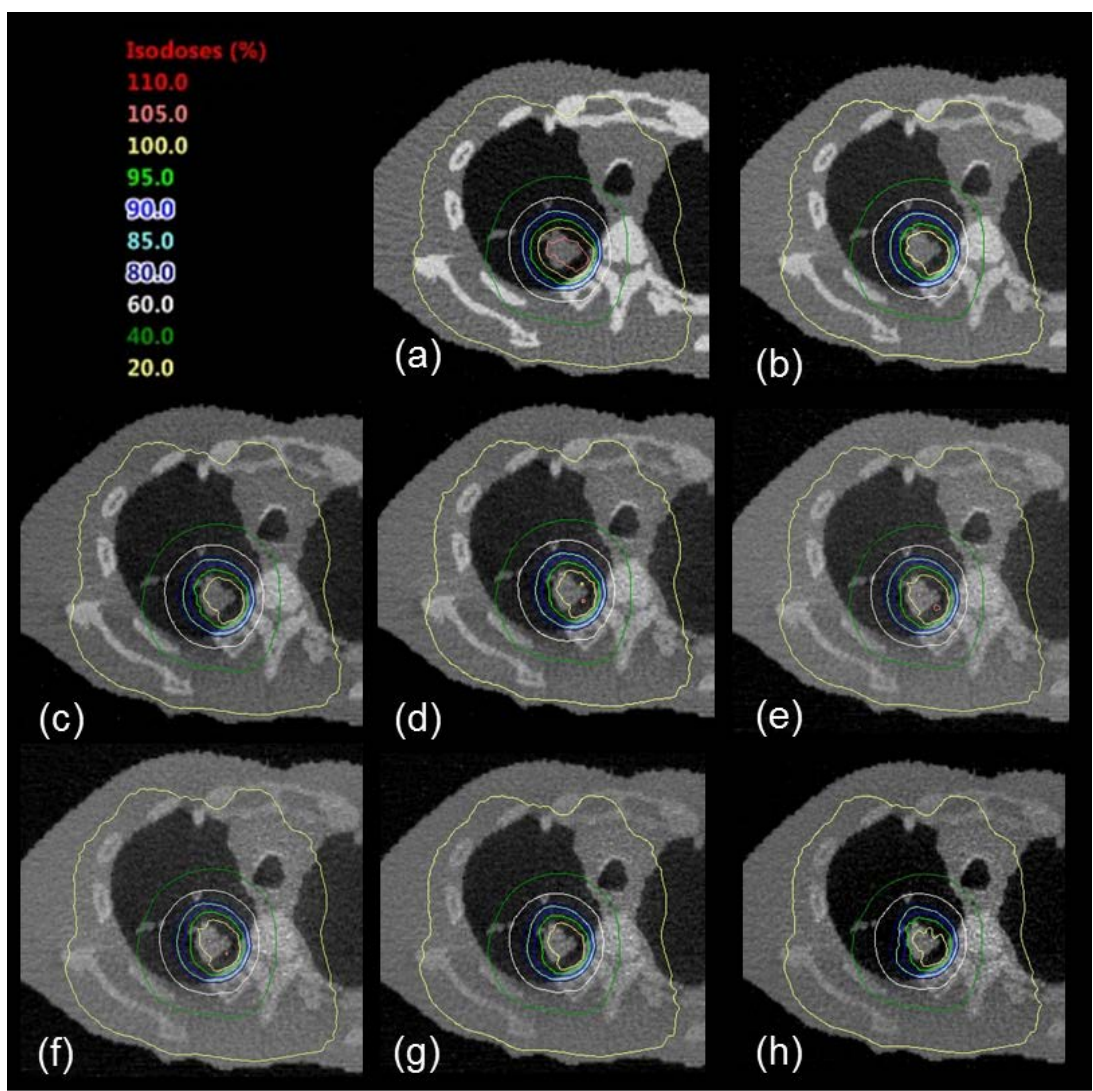

(B)

Figure 8. Dose distributions using the CBCT image with cupping artifact correction. (a): only primary photons, (b): $5 \%$, (c): $10 \%$, (d): $20 \%$, (e): $40 \%$, (f): $60 \%$, (g): $80 \%$, (h): $100 \%$ with scattered photons. (A): conventional five-field planning, (B): 1-arc VMAT planning. 


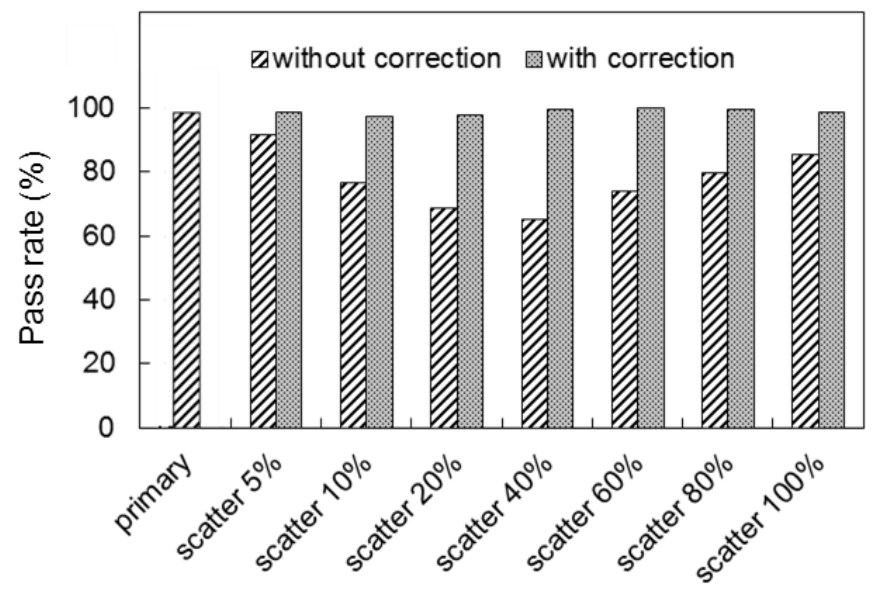

(A)

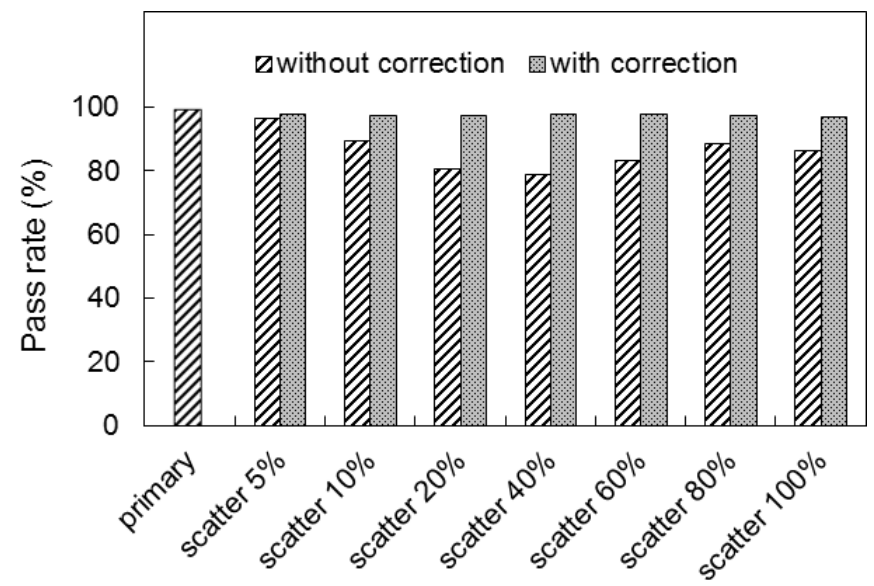

(B)

Figure 9. Results of the $\gamma$ analysis, (A) shows conventional five-field planning and (B) shows the 1-arc VMAT planning. The criteria for the pass rate were a $3 \%$ absolute dose and a $3-\mathrm{mm}$ distance agreement.

the dose distribution in the MSCT planning. The pass rate of the primary photon image was near $100 \%$; on the other hand, the pass rates of the images with scattered photons were significantly smaller than the pass rate of the primary photon image. Moreover, the pass rates of the images with cupping artifact correction were improved and did not depend on the amount of scattered photons.

Table 4 and Table 5 show the results of the DVH analysis for conventional and VMAT planning. In the primary photon image, the dose differences between each organ were similar to those in the MSCT planning; thus, the mean error rates of all organs were $<7 \%$. On the other hand, in the scattered photon images, the irradiated doses were reduced, and the error rates of the dose differences were increased to the negative side. For cupping artifact correction (Table 5), the dose differences and error rates for each organ were improved for all scattered photon images.

\section{Discussion}

A CBCT imaging device attached to a linear accelerator can be used for correct- 
Table 4. Dose differences without cupping artifact correction. Negative differences signify smaller values in the CBCT planning.

\begin{tabular}{|c|c|c|c|c|c|c|c|c|c|}
\hline & \multirow{2}{*}{ Parameter } & \multicolumn{8}{|c|}{ Error rate [\%] } \\
\hline & & primary & scatter $5 \%$ & scatter $10 \%$ & scatter $20 \%$ & scatter $40 \%$ & scatter $60 \%$ & scatter $80 \%$ & $\begin{array}{l}\text { scatter } \\
100 \%\end{array}$ \\
\hline \multirow{8}{*}{ Conventional } & PTV $D_{95}(\%)$ & 2.0 & -7.8 & -11.8 & -14.5 & -15.2 & -12.9 & -12.5 & -11.7 \\
\hline & PTV $D_{\text {mean }}(\%)$ & 0.0 & -8.1 & -11.9 & -14.4 & -15.2 & -13.0 & -12.3 & -11.5 \\
\hline & CTV $D_{95}(\%)$ & 1.5 & -8.4 & -12.5 & -15.0 & -15.9 & -13.7 & -13.4 & -12.9 \\
\hline & CTV $D_{\text {mean }}(\%)$ & -0.8 & -8.1 & -11.9 & -14.5 & -15.3 & -13.2 & -12.4 & -11.4 \\
\hline & Lung $V_{20}(\mathrm{~Gy})$ & -5.3 & -18.1 & -24.3 & -28.2 & -29.3 & -25.6 & -24.5 & -23.3 \\
\hline & Lung $V_{5}(\mathrm{~Gy})$ & -6.8 & -10.1 & -12.1 & -13.3 & -13.5 & -12.1 & -11.3 & -10.6 \\
\hline & Cord $D_{\mathrm{lcc}}(\mathrm{Gy})$ & -4.3 & -11.3 & -15.1 & -18.0 & -19.4 & -16.4 & -14.8 & -12.4 \\
\hline & Cord $D_{\text {mean }}(\%)$ & -3.8 & -9.2 & -13.0 & -15.7 & -17.3 & -14.6 & -13.0 & -10.8 \\
\hline \multirow{8}{*}{ VMAT } & PTV $D_{95}(\%)$ & 3.8 & -6.1 & -8.9 & -9.8 & -10.2 & -9.8 & -11.4 & -17.4 \\
\hline & PTV $D_{\text {mean }}(\%)$ & -0.5 & -7.6 & -10.5 & -11.7 & -11.9 & -11.0 & -10.9 & -12.7 \\
\hline & CTV $D_{95}(\%)$ & 0.6 & -7.7 & -10.5 & -11.9 & -12.7 & -11.3 & -11.5 & -14.2 \\
\hline & CTV $D_{\text {mean }}(\%)$ & -1.7 & -8.5 & -11.5 & -13.0 & -13.3 & -12.3 & -11.7 & -12.5 \\
\hline & Lung $V_{20}(\mathrm{~Gy})$ & -4.2 & -15.5 & -19.7 & -21.4 & -21.4 & -20.6 & -21.1 & -25.9 \\
\hline & Lung $V_{5}(\mathrm{~Gy})$ & -4.6 & -10.6 & -13.5 & -15.3 & -15.6 & -14.9 & -14.6 & -16.1 \\
\hline & Cord $D_{\mathrm{lcc}}(\mathrm{Gy})$ & -4.1 & -11.4 & -14.9 & -16.9 & -16.7 & -15.3 & -13.7 & -13.5 \\
\hline & Cord $D_{\text {mean }}(\%)$ & -2.5 & -10.0 & -13.4 & -14.9 & -14.9 & -13.4 & -11.9 & -11.4 \\
\hline
\end{tabular}

Table 5. Dose differences with cupping artifact correction. Negative differences signify smaller values in the CBCT planning.

\begin{tabular}{|c|c|c|c|c|c|c|c|c|}
\hline & \multirow{2}{*}{ Parameter } & \multicolumn{7}{|c|}{ Error rate [\%] } \\
\hline & & scatter $5 \%$ & scatter $10 \%$ & scatter $20 \%$ & scatter $40 \%$ & scatter $60 \%$ & scatter $80 \%$ & scatter $100 \%$ \\
\hline \multirow{7}{*}{ Conventional } & PTV $D_{95}(\%)$ & -3.3 & -4.0 & -3.3 & -1.8 & -1.6 & -2.4 & -4.4 \\
\hline & PTV $D_{\text {mean }}(\%)$ & -3.6 & -4.0 & -3.1 & -1.7 & -1.6 & -2.2 & -3.6 \\
\hline & CTV $D_{95}(\%)$ & -3.7 & -4.5 & -4.0 & -2.6 & -2.4 & -3.3 & -5.2 \\
\hline & CTV $D_{\text {mean }}(\%)$ & -3.7 & -4.1 & -3.3 & -2.1 & -2.0 & -2.4 & -3.4 \\
\hline & Lung $V_{20}(\mathrm{~Gy})$ & -11.6 & -11.8 & -10.9 & -8.6 & -8.5 & -9.9 & -12.0 \\
\hline & Lung $V_{5}(\mathrm{~Gy})$ & -8.7 & -9.5 & -9.3 & -8.7 & -8.8 & -9.2 & -9.3 \\
\hline & Cord $D_{1 c c}(\mathrm{~Gy})$ & -7.6 & -8.7 & -8.5 & -7.7 & -7.3 & -7.4 & -7.5 \\
\hline \multirow{9}{*}{ VMAT } & Cord $D_{\text {mean }}(\%)$ & -5.9 & -7.0 & -7.0 & -6.5 & -5.9 & -6.5 & -5.9 \\
\hline & PTV $D_{95}(\%)$ & -2.3 & -3.0 & -2.4 & -1.0 & -1.3 & -3.2 & -9.0 \\
\hline & PTV $D_{\text {mean }}(\%)$ & -4.0 & -4.5 & -4.2 & -3.4 & -3.4 & -4.3 & -6.2 \\
\hline & CTV $D_{95}(\%)$ & -4.0 & -5.2 & -5.1 & -3.9 & -4.2 & -5.7 & -9.2 \\
\hline & CTV $D_{\text {mean }}(\%)$ & -5.0 & -6.0 & -5.9 & -5.3 & -5.3 & -6.1 & -7.4 \\
\hline & Lung $V_{20}(\mathrm{~Gy})$ & -10.0 & -11.3 & -10.0 & -8.7 & -9.2 & -10.3 & -14.2 \\
\hline & Lung $V_{5}(\mathrm{~Gy})$ & -7.9 & -10.8 & -8.9 & -7.8 & -10.4 & -10.5 & -10.1 \\
\hline & Cord $D_{\mathrm{lcc}}(\mathrm{Gy})$ & -7.8 & -8.9 & -9.2 & -8.6 & -8.5 & -8.8 & -9.3 \\
\hline & Cord $D_{\text {mean }}(\%)$ & -6.5 & -7.5 & -8.0 & -7.0 & -7.0 & -7.5 & -8.0 \\
\hline
\end{tabular}


ing the patient set-up position, verifying a target position, and observing deformation of a tumor and normal tissues. However, a CBCT image is commonly degraded by scattered photons and thus, cannot be directly used for dose calculation. In this study, to evaluate the effect of the scattered photons for achieving CBCT-based dose calculation, we investigated the effect of scattered photons on calibration curves of CT values to electron densities and calculated doses by using the MC method.

Figure 3 shows the results of the reconstructed image of the ED phantom. In the primary photon image (Figure 3(i), Figure 3(ii)-(a)), each density material can be visually identified. However, as the contamination of the scattered photons increased, it was difficult to determine the position of each material density area, and cupping artifacts appeared on the reconstructed image with scattered photons. On the other hand, application of cupping artifact correction (Figure 3(ii)) showed that image uniformity significantly improved, but the visibility of these materials remained unclear. For the ED curves acquired from these ED phantom images, the dynamic range was smaller than that for the curve of the primary photon image, and this phenomenon was remarkable in the curves that included a high ratio of scattered photons. That is, the scattered photons in the ED phantom decrease the measured linear attenuation coefficients in the CBCT images. Moreover, the fluence of the scattered photon was depended on the location into the ED phantom, scatter-to-primary fluence ratio at the position of the center was higher than at the position of the edge in the ED phantom. Therefore, this heterogeneity due to position dependence of scattered fluence decreased the accuracy of the ED curves including the scattered photon, because locating the density materials in the center and the edge of the ED phantom. For the ED curve with cupping artifact correction (Figure 4(ii)), the pixel values near the center of the ED phantom image were corrected to the ideal pixel value of the water cylinder; therefore, ED curves with scattered photon contamination were closer to the ideal value (primary photon). Cupping artifact correction appeared to be an effective method for improving the accuracy of the ED curves from the ED phantom CBCT system images.

Figure 5 shows the image uniformity results. Image uniformity with scattered photons was degraded by approximately $32 \%$ from the primary photon image (Table 3). Image uniformity was most degraded $\left(t_{\text {cup }}: 37.7 \%\right)$ for images with a scattered photon ratio of $40 \%$ (Figure $5(\mathrm{e})$ ). In this result, because the fractionations of the pixel values caused by the scattered photons were increased, which is shown in the profile results for each image (Figure 5), differences in the mean pixel values of each ROI between the center and edge positions became small, and therefore calculated results of the uniformity were to be slightly smaller in the classes of $40 \%$ or more contamination of the scattered photon.

For the calculated dose distribution results in the lung image, the dose distribution in the primary photon image (Figure 7, Figure 8(a)) was similar to that in the MSCT image (Figure 6) with conventional and VMAT planning. On the other hand, the dose distributions using scattered photon images (Figures 
7(b)-(h)) differed from those in the MSCT planning in terms of the shape of the isodose curves near the tumor. Therefore, the $\gamma$ pass rates of the conventional and VMAT dose distributions in the scattered photon images were reduced, respectively, by 7.5, 3.6\% (scatter, 5\%), 22.7, 10.5\% (scatter, 10\%), 30.5, 11.9\% (scatter, 20\%), 33.8, 21.3\% (scatter, 40\%), 25.3, 16.7\% (scatter, 60\%), 19.4, 11.3\% (scatter, $80 \%$ ), and $13.813 .5 \%$ (scatter, $100 \%$ ) relative to those in the MSCT planning (Figure 6(A)). Moreover, the $\gamma$ pass rate of the dose distribution with scattered photons at a ratio of $40 \%$ was most decreased because of the degraded image uniformity. On the other hand, in the results of the dose distribution with cupping artifact correction shown in Figure 8, dose distributions near the tumor were similar to those in the MSCT planning, and these dose distributions did not depend on the amount of scattered photons. Therefore, results of the $\gamma$ pass rates with cupping artifact correction in the conventional and VMAT planning were significantly improved, respectively, by $0.5,2.3 \%$ (scatter, $5 \%$ ), 1.6, $2.6 \%$ (scatter, $10 \%$ ), $1.3,2.4 \%$ (scatter, $20 \%$ ), $-0.6,2.0 \%$ (scatter, $40 \%$ ), $-0.7,2.1 \%$ (scatter, $60 \%$ ), $-0.5,2.4 \%$ (scatter, $80 \%$ ), and $0.4,3.2 \%$ (scatter, $100 \%$ ) relative to those in the MSCT planning. In these results, the cupping artifact correction method was more favorable to the VMAT plan, because, the area of dose deviations caused by the cupping artifact was increased in the VMAT plan than these of the conventional plan (the VMAT plan irradiated large area of the patient body than the irradiated of conventional plan).

Table 4 shows the result of the DVH analysis without cupping artifact correction. In the primary photon image, results of the dose index in each organ were similar to those of the MSCT planning within the error rate of $\pm 7 \%$. However, in the scattered photon images, all organ dose indexes were reduced, and the error rates of the CTV mean doses in the conventional and VMAT planning with a scattered photon ratio of $40 \%$ increased by $15.3 \%$ and $13.3 \%$ more than those in the MSCT planning, for the worst case. The scattered photons decreased the measured linear attenuation coefficients in the CBCT images; therefore, the material densities in the lung $\mathrm{CBCT}$ images were assigned lower density values than those in MSCT planning. In Table 5, DVH results with cupping artifact correction showed that differences in the doses of all organs between MSCT planning and $\mathrm{CBCT}$ planning with correction were small; thus, the error rates of the CTV mean doses with scattered photons in the ratio of $40 \%$ were decreased by $2.1 \%$ and 5.3\% (conventional and VMAT planning) relative to those in the MSCT planning. Moreover, the error rates of the dose indexes for all organs were improved within approximately $10 \%$.

The CBCT images were strongly affected by scattered photons, and the calibration table of electron density was inaccurate. In the conventional planning, the relative electron density values from the pixel values in the CBCT image was used for the AAA dose calculation algorithm. In the VMAT planning, the mass density values from the pixel values in the CBCT images were used for the Acuros $\mathrm{XB}$ dose calculation algorithm. In both calculation algorithms, the calculation accuracies using the CBCT images with beam-hardening artifacts were de- 
graded; therefore, the quality of the CBCT images needed to be corrected to achieve CBCT-based dose calculation. To achieve highly accurate CBCT dose calculations, scattered photons contamination should be maintain by approximately $<5 \%$ of the total variation. Therefore, applying the scattered photon correction method and development of the anti-scatter grid for the CBCT system, which can block the scattered photons contaminating the detector, was useful for achieving CBCT-based ART. Moreover, because cupping artifact correction significantly improved dose calculation accuracy, this method was found to be an effective correction technique for dose calculations using CBCT images.

The influences of the fluctuation of MC calculation data were small for results of dose calculation with scattered photon contamination, since the accuracy of MC simulation within $1.0 \%$. In this study, objectives (ED phantom and lung images) assumed the use of uniform water material, and the ideal pixel value with a mono-beam spectrum and beam-hardening correction was calculated. If we assume the use of an object with several organ regions and calculate the ideal pixel value for each organ, the accuracy of the ED curve and dose distribution can be improved. However, accurate segmentation of each organ in the clinical $\mathrm{CBCT}$ images is difficult, because, the $\mathrm{CBCT}$ image have large fractionations by the scattered photon and motion artifact. And, inaccurate segmentation leads to cause wrong passing projection lengths and pixel values. Therefore, accurate segmentation method for improving the cupping artifact correction method in the $\mathrm{CBCT}$ image is needed for improving the accuracy of the ED curve and dose calculation.

\section{Conclusion}

We quantitatively analyzed the effect of scattered photons on dose calculations. The quality of $\mathrm{CBCT}$ images was strongly affected by scattered photons and should be corrected to achieve СВCT-based dose calculation. We also demonstrated that the accuracy of the CBCT-based dose calculation can be improved by removing scattered photons in approximately $<5 \%$ of the total variation and applying the cupping artifact correction method.

\section{References}

[1] Baymey, B.M., Lee, R.J., Handrahan, D., Welsh, K.T., Cook, J.T. and Sause, W.T. (2011) Image-Guided Radiotherapy (IGRT) for Prostate Cancer Comparing KV Imaging of Fiducial Markers with Cone Beam Computed Tomography (CBCT). International Journal of Radiation Oncology Biology Physics, 80, 301-305. https://doi.org/10.1016/j.ijrobp.2010.06.007

[2] Ding, G.X., Duggan, D.M., Coffey, C.W., Deeley, M., Hallahan, D.E., Cmelak, A. and Malcolm, A. (2004) A Study on Adaptive IMRT Treatment Planning Using KV Cone-Beam CT. Radiotherapy and Oncology, 85, 116-125. https://doi.org/10.1016/j.radonc.2007.06.015

[3] Schwartz, D.L. and Dong, L. (2007) Adaptive Radiation Therapy for Head and Neck Cancer-Can an Old Goal Evolve into a New Standard? Journal of Oncology, 2011, Article ID 690595. http://dx.doi.org/10.1155/2011/690595 
[4] Richter, A., Hu, Q., Steglich, D., Baier, K., Wilbert, J., Guckenberger, M. and Flentje, M. (2008) Investigation of the Usability of Conebeam CT Data Sets for Doe Calculation. Radiation Oncology, 3, 42. http://doi.org/10.1186/1748-717X-3-42

[5] Hu, W., Ye, J., Wang, J., Ma, X. and Zhang, Z. (2010) Use of Kilovoltage X-Ray Volume Imaging in Patient Dose Calculation for Head-and-Neck and Partial Brain Radiation Therapy. Radiation Oncology, 5, 29. http://doi.org/10.1186/1748-717X-5-29

[6] Rong, Y., Smilowitz, J., Tewatia, D., Tomé, W.A. and Paliwal, B. (2010) Dose Calculation on KV Cone Beam CT Images: An Investigation of the Hu-Density Conversion Stability and Dose Accuracy Using the Site-Specific Calibration. Medical Dosimetry, 35, 195-207. https://doi.org/10.1016/j.meddos.2009.06.001

[7] Fotina, I., Hopfgartner, J., Stock, M., Steininger, T., Lütgendorf-Caucig, C. and Georg, D. (2012) Feasibility of CBCT-Based Dose Calculation: Comparative Analysis of HU Adjustment Techniques. Radiotherapy and Oncology, 104, 249-256. https://doi.org/10.1016/j.radonc.2012.06.007

[8] Onozato, Y., Kadoya, N., Fujita, Y., Arai, K., Takeda, K., Kichi, K., Umezawa, R., Matsushita, H. and Jingu, K. (2014) Evaluation of on-Board KV Cone Beam Computed Tomography-Based Dose Calculation with Deformable Image Registration Using Hounsfield Unit Modifications. International Journal of Radiation Oncology Biology Physics, 89, 416-423. https://doi.org/10.1016/j.ijrobp.2014.02.007

[9] Huang, P., Yu, G., Chen, J., Ma, C., Yin, Y., Liang, Y., Li, H. and Li, D. (2017) Investigation of Dosimetric Variations of Liver Radiotherapy Using Deformable Registration of Planning CT and Cone-Beam CT. Journal Applied Clinical Medical Physics, 18, 66-75. https://doi.org/10.1002/acm2.12008

[10] Zhu, L., Xie, Y.Q., Wang, J. and Xing, L. (2009) Scatter Correction for Cone-Beam CT in Radiation Therapy. Medical Physics, 36, 2258-2268. https://doi.org/10.1118/1.3130047

[11] Maltz, J.S., Gangadharan, B., Bose, S., Hristov, D.H., Faddegon, B.A., Paidi, A. and Bani-Hashemi, A.R. (2008) Algorithm for X-Ray Scatter, Beam-Hardening, and Beam Profile Correction in Diagnostic (Kilovoltage) and Treatment (Megavoltage) Cone Beam CT. IEEE Transactions on Medical Imaging, 27, 1791-1810. https://doi.org/10.1109/TMI.2008.928922

[12] Sun, M. and Star-Lack, J.M. (2010) Improved Scatter Correction Using Adaptive Scatter Kernel Superposition. Physics in Medicine and Biology, 21, 6695-6720. https://doi.org/10.1088/0031-9155/55/22/007

[13] Thing, R.S., Bernchou, U., Mainegra-Hing, E. and Brink, C. (2013) Patient-Specific Scatter Correction in Clinical Cone Beam Computed Tomography Imaging Made Possible by the Combination of Monte Carlo Simulations and a Ray Tracing Algorithm. Acta Oncologica, 52, 1477-1483. https://doi.org/10.3109/0284186X.2013.813641

[14] Jarry, G., Graham, S.A., Moseley, D.J., Jaffray, D.J., Siewerdsen, J.H. and Verhaegen, F. (2006) Characterization of Scattered Radiation in kV CBCT Images Using Monte Carlo Simulations. Medical Physics, 33, 4320-4329. https://doi.org/10.1118/1.2358324

[15] Matsumoto, M. and Nishimura, T. (1998) Mersenne Twister: A 623-Dimensionally Equidistributed Uniform Pseudo-Random Number Generator. ACM Transactions on Modeling and Computer Simulation, 8, 3-30. https://doi.org/10.1145/272991.272995

[16] GAMMEX (2004) Tissue Characterization Phantom Model 467 User's Guide. GAMMEX, Middleton, WI. 
[17] Feldkamp, L.A., Davis, L.C. and Kress, J.W. (1984) Practical Cone-Beam Algorithm. Journal of the Optical Society of America A, 1, 612-619. https://doi.org/10.1364/JOSAA.1.000612

[18] Siewerdsen, J.H., Moseley, D.J., Bakhtiar, B., Richard, S. and Jaffray, D.A. (2004) The Influence of Antiscatter Grids on Soft-Tissue Detectability in Cone-Beam CT with Flat-Panel Detectors. Medical Physics, 31, 3506-3520.

https://doi.org/10.1118/1.1819789

Submit or recommend next manuscript to SCIRP and we will provide best service for you:

Accepting pre-submission inquiries through Email, Facebook, LinkedIn, Twitter, etc. A wide selection of journals (inclusive of 9 subjects, more than 200 journals) Providing 24-hour high-quality service User-friendly online submission system Fair and swift peer-review system Efficient typesetting and proofreading procedure Display of the result of downloads and visits, as well as the number of cited articles Maximum dissemination of your research work

Submit your manuscript at: http://papersubmission.scirp.org/ Or contact ijmpcero@scirp.org 